\title{
El mercado de la traducción de planta en Lima (Perú)
}

Fecha de recepción: 16 de noviembre de 2016 Fecha de aprobación: 22 de diciembre de 2016 Pp. 159-190
Sharon Escobedo Quintana Universidad Peruana de Ciencias Aplicadas sescobedo2393@gmail.com

Mijail Garvich Claux ${ }^{2}$

Universidad Peruana de Ciencias Aplicadas mgarvichclaux@gmail.com

Cómo citar este artículo: Escobedo, S. y Garvich, M. (2016). El mercado de la traducción de planta en Lima (Perú). Comunicación, cultura y política, 7, pp 159-190.

\section{Resumen}

En los últimos años, los servicios de traducción han cobrado mayor importancia, debido a la globalización y a las nuevas interrelaciones económicas. El mercado peruano no se encuentra exento a esta realidad, y los servicios de traducción han incrementado su demanda. Por tal motivo, se decide realizar esta aproximación hacia el estudio del mercado de la traducción de planta en Lima -Perú, cuyo foco estuvo puesto sobre los traductores profesionales. Planteado como un estudio de caso, el siguiente texto se elabora a partir del contraste de información recolectada a través de una serie de entrevistas y algunos estudios previos al respecto y tiene por objetivo comprender la situación del traductor de planta en la actualidad: las ventajas y desventajas de su labor y los desafíos que debe enfrentar.

\section{Palabras clave}

Traducción de planta, lenguas, empresas, estatus del traductor.

\footnotetext{
${ }^{1}$ Bachiller en Traducción e Interpretación Profesional de la Universidad Peruana de Ciencias Aplicadas.

${ }^{2}$ Bachiller en Ciencias de la Comunicación, Universidad Inca Garcilaso de la Vega, Lima, Perú (2002). Licenciado en Producción de Cine, Radio y Televisión, Universidad Inca Garcilaso de la Vega, Lima, Perú (2005). Maestro (c) en Psicología, Universidad de San Martín de Porres, Lima Perú. ORCID: https://orcid. org/0000-0002-0773-8855
} 


\title{
The market for translation in Lima (Peru)
}

\begin{abstract}
In recent years, translation services have become more important because of the globalization and new economic interrelationships. The Peruvian market is not exempt from this reality, and the translation services have increased their demand. For this reason, we decided to make this approach towards the study of the translation market in LimaPeru, whose focus was placed on professional translators. Staked out as a case study, the following text is generated based on the contrast of information that was collected through a series of interviews and some previous studies in this respect, and aims at understanding the situation of the full time translator today: the advantages and disadvantages of their work and the challenges they must face.
\end{abstract}

\section{Keywords}

Full time translation, languages, enterprises, translator status.

\section{Le marché de la traduction à Lima (Pérou)}

\section{Résumé}

Les services de traduction ont retrouvé ces dernières années de leur importance passée du fait de la globalisation et du rebond de l'activité économiques. Le marché péruvien s'ancre dans cette réalité et les services de traduction se sont accrus. Cet article réalise une étude du marché de la traduction professionnelle de Lima-Pérou. Construit sur le schéma d'une étude de cas, ce texte est d'abord élaboré à partir d'informations récoltées au travers d'une série d'entretiens et d'études préalables mais aussi par le biais d'une compréhension objective de la situation actuelle du traducteur: avantages et inconvénients de son travail, défis auxquels il doit faire face.

\section{Mots clés}

Traduction, langues, entreprises, statut social du traducteur. 


\section{O mercado da tradução em Lima (Perú)}

\section{Resumo}

Nos últimos anos, os serviços de tradução tornaram-se mais importantes devido à globalização e as novas inter-relações econômicas. O mercado peruano não está isento a esta realidade e a demanda por serviços de tradução têm aumentado. Por esta razão, decide-se realizar um estudo de mercado de tradução em Lima-Peru, cujo foco foram os tradutores profissionais. Concebido como um estudo de caso, o seguinte trabalho foi elaborado a partir do contraste da informação coletada através de uma série de entrevistas e alguns estudos anteriores sobre o assunto e tem como objetivo compreender a situação do tradutor no momento: as vantagens e desvantagens de seu trabalho e os desafios que enfrentam.

\section{Palavras-chave}

Tradução, línguas, empresas, status do tradutor. 


\section{Introducción}

urante los últimos veinte años, el desarrollo de la economía peruana ha crecido de manera considerable (Ministerio de Trabajo y Promoción del Empleo, 2001) al convertirse en una de las economías más estables y prometedoras de la región (ASBANC, 2014). La inversión privada, en su mayoría, proveniente de países no hispanohablantes, incrementó la necesidad de más servicios de traducción en diversos ámbitos (Mora e Infante, 2012). Así es como, a partir de la década de 1990, un flujo considerable de capital extranjero se asentó en territorio nacional y, años más tarde, a través de la implementación de políticas neoliberales y la firma de tratados de libre comercio, la necesidad de contar con traducciones se hizo cada vez mayor (Castillo, Montoro y Tue, 2006). Los traductores, en consecuencia, tienen varios ámbitos en los que pueden trabajar, no solo como freelance, sino también como traductores de planta o de in-house, ya sea en empresas mineras, industriales, médicas, entre otras.

A partir de esta situación, surge la necesidad de conocer el mercado de traducción de planta, cuál es la oferta y la demanda, cuáles son las lenguas más requeridas, entre otros aspectos. Si se realiza una investigación sobre el mercado de la traducción planta, se puede evidenciar que múltiples países que han realizado investigaciones importantes. En España, por ejemplo, se tiene un estudio sobre el mercado de la traducción, donde se refiere que sólo un $15 \%$ de las empresas opta por contratar a un traductor de planta, dado que existe una tendencia a derivar sus encargos a agencias de traducción (Lozano, 2011). Entonces, surge la interrogante, ¿quién es un traductor de planta? Anna Kuznik (2010) alude a la investigación realizada por Dam y Zethsen, quienes definen al traductor de planta o traductor interno como un traductor formado profesionalmente, que tiene un contrato laboral establecido y cuya actividad principal dentro de la empresa sea traducir. Sin embargo, en la práctica, se sabe que muchos traductores empíricos laboran como traductores de planta (Franco y Quiroz, 2013). Por otro lado, en Colombia, Carlos Bello (2011) desarrolló una investigación titulada «Traducción empresarial en el mundo globalizado de hoy» que se relaciona con los perfiles de los traductores en ese país. En un pequeño acápite, el autor alude al trabajo del traductor de planta, al que define como el traductor que trabaja en el staff de una empresa o institución y además afirma que la combinación de lenguas de mayor demanda es la de inglés- 
español, y que existe una mayor demanda más traducciones directas (hacia la lengua materna) que inversas (hacia la lengua extranjera). En el mismo país, Clavijo et al. (2006) presentaron un estudio de 34 empresas transnacionales instaladas en Colombia que cuentan con servicios de traducción de planta. El estudio se enfoca, entre otros aspectos, en las características que todo traductor de planta debe tener en un contexto globalizado.

Luego de referir los contextos de estudios internacionales sobre la traducción de planta, se puede afirmar que en Perú no se cuenta con un estudio que se enfoque en esta temática. Hasta el momento, solo se ha realizado una investigación no publicada sobre el mercado de la traducción (Benavides, Gálvez, Villanueva y Garvich, 2015)., sin embargo, estos autores no se enfocaron en la traducción de planta en empresas que no son agencias de traducción.

Por ello, el objetivo del estudio de caso fue conocer cuál era la situación actual del mercado de la traducción de planta, tanto para profesionales de la traducción que se encuentran inmersos en esta actividad, como para las empresas que contratan este tipo de servicio, y comparar los resultados obtenidos con otros estudios poder brindar así una primera aproximación del mercado de la traducción de planta en Lima, Perú. Asimismo, se considera que la investigación ayudó a conocer cuáles son las actividades realizadas por los traductores de planta, las lenguas de trabajo, la demanda de traducciones y si existen otras actividades que el traductor realice dentro de la empresa. Esta información es útil no solo para los traductores egresados, sino también para aquellos que deseen insertarse en el mercado laboral de la traducción de planta al terminar la universidad, dado que contarán con una visión de los requerimientos que se exige en este ámbito. Del mismo modo, se considera que el estudio es útil para posteriores investigaciones que se enfoquen en la labor de los traductores de planta como parte del mercado laboral de la traducción y así aportar nuevos conocimientos sobre el mercado de la traducción en el Perú. 


\title{
2. Perspectivas internacionales sobre la traducción de planta
}

\begin{abstract}
continuación, se referirán algunas investigaciones en las que se describe A la situación y el trabajo del traductor de planta en distintos países de América, Europa y Asia. Esta revisión tiene como finalidad establecer los antecedentes y las posteriores discusiones de la presente investigación.
\end{abstract}

\subsection{Contexto latinoamericano}

En México, una investigación sobre la enseñanza del lenguaje técnico petrolero para la Licenciatura en Idiomas alude al papel que desempeñan los traductores de planta en este país, y, sobre todo, en el sector petrolero, dado que existe una gran demanda de traducciones de la combinación inglés-español (Alejandro, 2002). Respecto de los traductores de planta que trabajan dentro de las plantas petroleras, se refiere que muy pocos son traductores profesionales, la mayoría de ellos son ingenieros que tienen un dominio del inglés, y que por ello desempeñan la labor. La investigación refiere también que la lengua con mayor número de traducciones es el inglés, y que existen dos tipos de traductores: los que no tienen una formación universitaria como tal, y los que siguen una carrera para ser licenciados en Traducción (Alejandro, 2002). Asimismo, esta autora alude al escaso número de traductores especializados dentro de una empresa petrolera, dado que no hay instituciones educativas que brinden una especialización para los traductores, quienes se encuentran en desventaja frente a los conocimientos técnicos que sí tienen los especialistas. De este modo, al competir con los especialistas, los traductores tienen la posibilidad de perder puestos de trabajo o áreas de inserción laboral (Alejandro, 2002).

\subsection{Contexto norteamericano}

Por otro lado, en un estudio anual denominado Survey of the Canadian Translation Industry (2014), realizado en Canadá, se indica que la traducción de planta representa $30 \%$ de los servicios de traducción, por lo cual es un ámbito de trabajo representativo. Esta situación se genera a partir del bilingüismo 
existente en Canadá. Del mismo modo, se alude a la tendencia al alza de la contratación de traductores in-house, lo cual trae a colación la necesidad de obtener grados académicos, es decir, formar traductores profesionales y luego analizar cómo estos grados influyen en las cotizaciones y los pagos (Survey of the Canadian Translation, 2014).

\subsection{Contexto europeo}

Por otro lado, Kuznik (2011) menciona la hibridez y heterogeneidad del trabajo realizado por el traductor de planta. La autora se refiere al estudio realizado en Francia por la Société française des traducteurs, que contó con 42 participantes, 37 mujeres y 5 hombres, todos traductores egresados de Ecole supérieure d'interprètes et de traducteur. En este estudio, se evidenció que solo $49 \%$ del tiempo laboral correspondía a traducir, y que el restante, el $51 \%$, se orientaba a realizar otras actividades como corrección, gestión de subcontratación, trabajos terminológicos y también interpretaciones (Kuznik, 2011).

Asimismo, en otro estudio francés, se menciona que muchos traductores optan por trabajar en una compañía como traductores de planta, porque se enfocan en una producción técnica orientada hacia los negocios. Por otro lado, se alude a que el traductor de planta debe desempeñar un trabajo óptimo en comparación con un traductor que trabaja en una agencia, dado que tiene todos los recursos a su disposición (Gouadec, 2007).

\subsection{Contexto asiático}

Para el caso coreano, se tiene a Choy y Lim (2000), quienes señalan la diversidad de actividades realizadas por los traductores de planta y el trabajo continuo con agencias de traducción externas, es decir, hay una fusión de servicios entre los servicios internos de traducción y las agencias. Asimismo, respecto de la lengua coreana, los traductores tienen una considerable labor de corrección de traducciones inversas realizadas por traductores no nativos. 


\section{Métodos}

Dara este estudio, se realizó una investigación exploratoria de tipo cualitativo.

Se exploró el mercado de la traducción de planta desde la perspectiva de los traductores, dado que estos participantes fueron la principal fuente de información para el estudio. La investigación tuvo como objetivo describir las experiencias de aquellos traductores que decidieron trabajar en empresas que no son agencias de traducción y que tampoco optaron por trabajar por cuenta propia y que por ello forman parte de un ámbito laboral diferente.

Para llevar a cabo la investigación, se realizaron entrevistas a profundidad y orientadas por una guía estructurada, lo que permitió recolectar información pertinente. Las entrevistas tuvieron una duración mínima de 30 minutos y las preguntas se enfocaron en temáticas sobre la percepción de cada participante sobre el rol que desempeñaba dentro de la empresa donde laboraba, si creía que era valorado como traductor o si se reconocía la labor que realizaba. Asimismo, la investigación se complementó con encuestas breves de 9 preguntas, que se entregaron a los entrevistados luego de la culminación de las entrevistas. Estas se orientaban más a la escala salarial, a la combinación lingüística o a los tipos de documentos que traducían con mayor frecuencia.

Por otro lado, se realizó una triangulación de datos, a través de la comparación de toda la información obtenida de las entrevistas y encuestas, con un gran número de estudios previos sobre la misma temática, pero en diversos contextos. Se encontraron similitudes y diferencias que podrían considerarse como patrones para el trabajo de planta, sin embargo, se debe tomar en cuenta que esta investigación es una aproximación exploratoria y no se puede realizar generalizaciones.

\subsection{Población y contexto}

La población de estudio se localizó en Lima, Perú. Lima es la capital de Perú y la ciudad más poblada del país y eje central de la economía nacional (Gonzales de Olarte, 2001). Gran parte de las empresas transnacionales tienen sus oficinas centrales en esta ciudad. Para el estudio, se buscó trabajar con traductores de planta, egresados de la carrera de Traducción e Interpretación de 
las universidades UNIFÉ y Ricardo Palma, dado que estas instituciones tienen una mayor trayectoria y su número de egresados es mayor, en comparación a las otras instituciones.

La muestra estuvo conformada por 14 participantes que fueron elegidos con la ayuda de algunos docentes de la Carrera de Traducción e Interpretación Profesional de la Universidad Peruana de Ciencias Aplicadas que actuaron como gatekeepers. A través de su participación, se logró establecer citas o reuniones con los participantes que fueron 11 fueron mujeres y 3 varones. La edad de los participantes fluctuó entre los 25 y 50 años. Los sectores empresariales a los que pertenecieron fueron diversos. Hubo participantes del área minera e industrial, como también de laboratorios médicos, del sector educativo o de estudios de propiedad intelectual.

\subsection{Criterios de selección}

Para el reclutamiento de los participantes, se tomó en cuenta los siguientes criterios: ser licenciado de Traducción e Interpretación de la Universidad Ricardo Palma o de la Universidad Femenina del Sagrado Corazón y tener como mínimo un año de experiencia como traductor de planta desde el año 2012 hasta la fecha de recolección de datos. Si bien los criterios de selección fueron amplios y podían brindar la posibilidad encontrar a un gran número de participantes, fue muy difícil contactarlos y lograr que participen del estudio. No se les pudo contactar al ir a una empresa, sino que todo acercamiento con los participantes se dio a través de la red de contactos de los gatekeepers. Por otro lado, una de las dificultades con las que se tuvo que lidiar al principio fue que algunos de los potenciales candidatos a participantes se consideraban traductores de planta, pero en realidad eran traductores freelance que trabajaban para empresas a través de recibos por honorarios o facturas.

\subsection{Muestreo}

El muestreo fue no probabilístico, dado que los participantes no fueron elegidos al azar, sino que se les seleccionó de la población que se fue contactando. El tipo de muestreo no probabilístico fue propositivo, dado que los participantes 
fueron elegidos por los autores, en este caso, solo se eligió a traductores profesionales y se excluyó a los traductores empíricos o sin la experiencia mínima requerida. Por ello, el muestreo fue también homogéneo, puesto que los entrevistados compartieron características similares como formación académica, lenguas de trabajo y nacionalidad.

\subsection{Recolección de datos}

El principal método utilizado para la realización de esta investigación fue el uso de entrevistas que permitieron construir un panorama del mercado de la traducción de planta en Lima, a partir de las experiencias de los participantes. No se planteó un único espacio para realizar las entrevistas, dado que se consideró pertinente que los participantes eligieran el lugar según horario y disponibilidad.

Se propuso la utilización de entrevistas estructuradas, dado que ellas brindan la posibilidad de profundizar en los temas que se estudian. Se utilizó una guía estructurada que fue validada por una especialista en el tema. Las preguntas propuestas se enfocaron en temas como la combinación lingüística, es decir, las lenguas con las que trabajan los traductores; la valoración del trabajo como traductor de planta, es decir, el trato que reciben los traductores en sus lugares de trabajo; o la remuneración que perciben, entre otros. Por otro lado, la duración mínima de las entrevistas fue de treinta minutos y se necesitó una grabadora para recopilar la información.

Para complementar el estudio cualitativo, se llevaron a cabo encuestas breves que se centraron en los sectores empresariales que contratan a traductores de planta, las competencias requeridas para desempeñarse en dichos ámbitos, entre otros.

\subsection{Plan de análisis}

El plan de análisis se dio en una sola fase: se realizaron las respectivas transcripciones de cada una de las entrevistas, los resúmenes y se codificó las citas más representativas con el software Dedoose. Asimismo, se creó una base 
de datos en Excel donde se recopiló la información de las encuestas. Luego, a partir de las citas extraídas y codificadas, se procedió con la categorización de 2 temas y 3 subtemas, donde se presentan los principales resultados obtenidos. Después, se consultó la bibliografía recopilada previa a la investigación y se comenzó con la comparación de los resultados con otros autores, con lo que se logró crear una aproximación del estudio de caso de la traducción de planta en Lima, Perú.

\section{Resultados}

a tabla 1 refiere los datos obtenidos a través de las encuestas realizadas $\smile$ durante los meses de abril-agosto de 2016, y de las que se logró recopilar información relacionada con el sector de pertenencia, contrato y años de experiencia.

Tabla 1. Participantes y características

\begin{tabular}{|l|l|l|c|}
\hline Participante & Sector al que pertenece & Tipo de contrato & $\begin{array}{c}\text { Años de } \\
\text { experiencia }\end{array}$ \\
\hline Marta & Consultoría & Indefinido & 4 años \\
\hline Romina & Consultoría & Indefinido & 4 \\
\hline Juan & Educación & Indefinido & 4 \\
\hline Marcela & Industrial & Indefinido & 4 \\
\hline Ana & Laboratorios farmacéuticos & Por menos de seis meses & 4 \\
\hline Carmen & Minería & Indefinido & 4 \\
\hline María & Industrial & Indefinido & 5 \\
\hline Martín & Comunicaciones & Indefinido & 6 \\
\hline Carolina & Consultoría & Se seis meses a un año & 6 \\
\hline Reyna & Laboratorios farmacéuticos & De seis meses a un año & 6 \\
\hline Julia & Tecnología & Definido por más de un año & 6 \\
\hline Karina & Consultoría & Indefinido & 7 \\
\hline Silvia & Educación & Indefinido & 20 \\
\hline Pedro & Propiedad intelectual & De seis meses a un año & 23 años \\
\hline
\end{tabular}

Fuente. Elaboración propia. 


\subsection{Perfil del traductor de planta}

\subsubsection{Motivación para ser traductor}

Respecto de la motivación que llevó a los catorce participantes a elegir la Carrera de Traducción e Interpretación Profesional, todos refirieron el interés previo que tuvieron hacia el aprendizaje de lenguas extranjeras. Algunos estudiaron en colegios bilingües o asistieron a institutos de idiomas, lo cual luego les favoreció cuando comenzaron a estudiar la carrera profesional, porque ya solo debían enfocarse en aprender a traducir y ya no en aprender lenguas nuevas.

"En realidad, aproveché un poco que ya supiera el inglés y como que por ese lado lo exploté más. Me pareció algo que podía utilizar y que finalmente me iba a ayudar mucho en la carrera en sí”. (Karina)

Sin embargo, el gusto por las lenguas no fue la única motivación que estuvo presente, sino que hubo casos en los que existieron motivaciones personales, por ejemplo la influencia de algún familiar que se desempeñaba como traductor o como profesor de idiomas y fueron quienes los encaminaron hacia esta carrera.

"Pues no conocía la carrera y me comentaron sobre ella, una tía que había estudiado Traducción e Interpretación. Me dijo la diferencia entre estudiar idiomas y estudiar la carrera como tal. Me comentó, me llamó la atención y así me decidí”. (Carmen)

Si bien no se ha encontrado estudios que reflejen motivaciones tan personales por parte de los traductores para justificar su elección para convertirse en traductores, se puede afirmar que la mayoría de estudiantes, al iniciar la carrera, no busca una recompensa económica o material, sino que lo hacen por gusto o dedicación (Rodríguez y Sierra:2016). 


\subsubsection{Cómo llegó a ser traductor de planta}

El primer contacto que tuvieron los participantes con la traducción de planta se dio durante el periodo de prácticas preprofesionales o profesionales, mientras estaban en la universidad o apenas habían acabado la carrera. En el siguiente extracto, una participante refiere cómo ingresó a trabajar en una de las cuatro firmas de consultoría más importantes del mundo.

"Luego, se presentó la oportunidad de entrar como practicante de traducción en otra Big Four. Entonces, postulé, como ya conocía la temática, me fue muy fácil entrar a esta otra Big Four como practicante de traducción". (Romina)

Muchos de los traductores ingresaron a las empresas como practicantes, fue el primer contacto con el entorno o ámbito laboral (Kuznik, 2010). Las prácticas profesionales les brindan a los estudiantes o egresados la oportunidad de reconocer e identificar cómo es el mercado real, cuáles son las demandas de las empresas y así pueden aplicar todos los conocimientos que ha adquirido en su formación académica.

La manera en la que los participantes llegaron a ser traductores de planta fue a través de sugerencias de terceros como familiares o amigos y por medio de anuncios en los periódicos que solicitaban traductores.

"A mí me pasaron la voz porque mi papá tenía amigos que conocían a Adolfo Cunyas que es quien trabaja ahí en $\mathrm{PwC}$, y me enteré que estaban necesitando gente para traducir". (Marta).

"Bueno, un amigo me contactó, en realidad. Él entró primero a esta empresa a practicar y luego de unos meses me comentó que se estaba abriendo una plaza también para practicante y empecé allí como practicante". (Martín).

"Luego termino la carrera, y de ahí, revisando El Comercio [periódico peruano] nuevamente, yo he tenido una suerte increíble, gracias a Dios, vi un anuncio que pedían una traductora para la empresa Albis". (Reyna). 
No obstante, se evidenció un caso en el que un participante ingresó como reemplazo de un colega, pero terminó asumiendo el cargo de traductor de planta de la empresa.

"Me llamaron para hacer el reemplazo de una chica con una interpretación para unas entrevistas y me terminé quedando y, desde ese momento, estoy trabajando tanto en la parte de traducción como en la de interpretación". (Carmen)

De toda la bibliografía recopilada, no se logró encontrar alguna información que refiere cómo los traductores egresados se insertan en el mercado. La mayoría de investigaciones se enfocan en los sectores de inserción, la cantidad de profesionales que se encuentran laborando y si ejercen la carrera que estudiaron, en este caso, Traducción e Interpretación.

\subsubsection{Situación del traductor de planta}

Por otro lado, los resultados obtenidos y relacionados con la situación del traductor de planta revelan que aún no se tiene conocimiento pleno sobre la profesión de traductor-intérprete en Lima (Perú). Todos los participantes refirieron que, cuando un traductor ingresa a trabajar a una empresa, se enfrenta a concepciones erróneas sobre la profesión.

"En la empresa en la que trabajo, hay gente que no sabe quiénes son los traductores, hay gente que no sabe que existe el área de traducción, hay gente que no sabe que hay traductores ahí, somos invisibles". (Romina)

Como se puede observar, si bien la traducción es una actividad que existe desde hace muchos años, es aún muy joven como una actividad laboral o profesional, y se le considera como una actividad complementaria o simple (Franco y Quiroz, 2013). Al igual que esta participante, varios de los entrevistados refirieron que no hay un conocimiento pleno de lo que implica ser un traductor y de su importancia dentro de las empresas. Por ejemplo, al principio, se le suele confundir con alguien que solo sabe inglés o con secretarias bilingües. 
"La carrera de Traducción en muchas empresas, me parece que están mal informados y lo que buscan es una traductora-secretaria, una traductora con labores administrativas, que, en realidad, quitan mucho, desmeritan un poco la labor de un traductor profesional". (María).

Si bien el presente estudio no se centra en la perspectiva de los directivos o jefes de las empresas sobre la traducción de planta, existe un estudio en España, donde si bien la imagen del traductor es positiva, los empleadores desconocen qué implica en realidad ser un traductor, cuál es la labor que debe desempeñar, lo que trae consigo que no aproveche al máximo los servicios que los mismos le pueden ofrecer (Medina y Álvarez, 2014).

Asimismo, varios participantes refirieron la inexistencia de un área determinada, como una oficina para los traductores, y por ello muchos comparten el mismo espacio con otros profesionales, lo cual implicaría que no se les identifique como trabajadores de un área determinada de la empresa.

"Ni siquiera somos el área de traducción. Somos el área de inglés para ellos, va por ahí, no nos dan mucha importancia, tanto como darnos un lugar, e incluso, en los ingresos que percibimos, a veces creo que no van acorde con el mercado internacional”. (Martín).

"O sea dentro del organigrama de la empresa, ni siquiera es considerada como un área. Nosotros estamos metidos por ahí, dentro del área administrativa, pero no estamos considerados dentro del organigrama". (Romina).

Esta última cita concuerda con lo presentado por Kuznik (2010), quien alude a la investigación realizada por Hermans y Lambert, quienes afirman que el estatus del traductor de planta es bajo y que incluso sus traducciones son consideradas simples. Por ello, no hay oficinas del área de traducción de la empresa y no hay una proximidad física con los jefes y directivos.

Es entonces cuando surge un concepto que fue muy repetitivo en todos los participantes: se requiere educar al "cliente", indicarle cuáles son las funciones de un traductor y demostrar que son necesarios, y que no se debe 
desvalorizar su trabajo, al creer que solo se requiere conocer lenguas. Así, luego de cambiarles la perspectiva que tienen sobre la profesión dentro de la empresa, se generan cambios sustanciales.

"Pero cuando ya la conocen (a la carrera), cuando tienes la oportunidad de explicarles, de contarles un poco de qué se trata tu carrera, tu profesión, ellos la entienden, y la valoran muchísimo". (María).

Esa valoración coincide mucho con la presentada por Kuznik respecto a la percepción sobre el traductor de planta dentro de una empresa (2016):

"He is presented as a qualified, competent, multi-tasking, independent, involved (committed) and active worker. He is an employee who, with a great deal of patience and expertise, participates in the production process and takes responsibility for the results." (Kuznik, 2016, p 229).

Estos resultados fueron interesantes, dado que, en otra investigación, realizada por Dam y Zethsen, se afirma que los traductores con menor años de experiencia consideran que la valoración del traductor in-house es mucho mayor a la que tienen los traductores con mayores años de experiencia (Kuznik, 2016) y en el presente estudio de caso es lo contrario. Los traductores con menor número de años de experiencia consideran que el trato no es tan favorable como los que cuentan con más años de trabajo como traductor de planta.

Por otro lado, la temática de las relaciones laborales fue representativa. Los participantes refirieron que en sus empresas existen buenos climas laborales, pero que las principales dificultades que pueden generar algún tipo de malestar se encuentran en los plazos de entrega, la cantidad de documentos para traducir y el hecho de trabajar con personas de diversas profesiones.

"Con mis compañeros, siempre me he llevado bien, manejando también los puntos álgidos. Siempre los puntos álgidos van a ser los plazos de entrega: quieren rápido las traducciones, entonces, ahí tienes que tener un manejo de arte de negociación y hacerles ver que no es tan rápido como ellos creen”. (Reyna). 
"En realidad, es superar el momento porque a veces, uno tiene muy poco tiempo, generalmente es para los traductores, el peor obstáculo que superar es el tiempo". (Pedro).

Una vez más a Dam y Zethzen, en el artículo de Kuznik, afirman que los traductores de su estudio si lograron integrarse y compenetrarse con el resto del personal de la empresa, Además, al haber logrado este clima laboral adecuado, sentían que su estatus laboral era mejor (Kuznik, 2010). Los participantes consideraron el tiempo como un factor preponderante en la entrega de traducciones, los encargos de diferentes áreas podían aparecer en un solo momento e incluso tener la misma fecha de entrega. Por ello, explicarle a los compañeros y jefes que la traducción toma tiempo y que no solo se traduce para un área en específico, evita conflictos con los colegas de otras áreas.

\subsection{La labor del traductor de planta}

\subsubsection{Competencias del traductor de planta}

En lo que concierne a las competencias que todo traductor de planta debe tener, los participantes consideraron que la competencia básica es la lingüística, aquella que refiere al dominio de las lenguas con las que se trabaja. Todos concordaron que se debe tener un dominio total de la lengua materna y de la lengua extranjera (para todos los participantes la combinación es inglésespañol y el español es la lengua materna).

"Yo diría que el traductor de planta, primero lo que tiene que manejar, lo cual todo traductor debe conocer es la competencia lingüística, lo cual te enseñaron en la universidad". (Reyna)

"Tú tienes que tener un manejo de la lengua materna y de la lengua a la que vas a traducir, y claro, depende de la dirección en la que vayas a traducir". (Natalia)

Márquez (2013), refiere al Grupo PACTE, y considera como competencia primordial a la competencia comunicativa bilingüe, al conocimiento de las lenguas con las que trabaja el traductor y todas las implicancias del 
conocimiento lingüístico, semántico, morfológico y lexical. Para todos los participantes esta competencia fue considerada como la fundamental, y que la debería de tener un mayor desarrollo y dominio.

En segundo lugar, se refirió a la competencia de relaciones interpersonales y de control de emociones, dado que, al trabajar en una empresa con muchos perfiles profesionales, suele haber ciertos momentos de tensión que se encuentran en los plazos de entrega y la carga laboral. Los traductores tienen que aprender a controlar sus emociones, contestar de la mejor manera a los reclamos y, sobre todo, lidiar con el estrés. Hay temporadas en las que las demandas de traducciones aumentan sustancialmente y, por ello, los traductores no se dan abasto. Pero, luego, hay temporadas en los que el número de traducciones disminuye.

"Y tienes que saber cómo tomarlo, porque si eres de esas personas que se abruman por el estrés, por la carga laboral, y de pronto ya no sabes qué hacer, lo tomas mal, puede que reacciones mal, puede que le conteste feo, porque te agarró en un momento que no era tu momento". (Marta).

Esta competencia a la que se refieren los participantes es la de flexibilidad, la que permite que el traductor se pueda adaptar y lidiar con diferentes entornos y personas (Márquez: 2013). El trabajar con diferentes perfiles profesionales implica una gran interacción interpersonal, y, por ejemplo, si no se logra un consenso sobre los plazos de entrega, o no se les explica a los compañeros que traducir no es tan fácil como parece, el clima laboral puede ser un obstáculo para el buen desempeño del traductor.

Asimismo, los participantes mencionaron la competencia estratégica y de organización que implica ser muy ordenado con los documentos, organizarlos de la mejor manera para llevar un control óptimo.

"Creo que también una competencia importante para las traductoras es ser ordenada y organizada y cuidadosa, cuidar bastante los detalles". (Carolina). 
Según Márquez, los traductores necesitan desarrollar las competencias de atención al detalle y la competencia de orientación a la calidad, exactitud y orden (Márquez, 2013). Estas competencias son necesarias, dado que permiten que el traductor realice un trabajo con mayor control, eficacia y eficiencia.

De otro lado se tiene que para los traductores, es importante también aprender a trabajar de acuerdo con los horarios que imponen las empresas y negociar los plazos de entrega. Hay participantes que trabajan de lunes a viernes, ocho horas diarias, y otros que trabajan de lunes a sábado.

"Tienes que ser negociador, tú negocias los plazos de entrega con tu jefe, con tus compañeros, con el personal de otras áreas". (Reyna)

\subsubsection{Actividades que realiza el traductor de planta}

Dentro de las empresas, los traductores realizan diversas labores que abarcan desde la recepción de mensajes y llamadas hasta interpretaciones. Los traductores se encargan de recepcionar las llamadas y contestar los mensajes cuando estos son en inglés, dado que muchos de los trabajadores de la empresa no saben el idioma o no se sienten seguros de hablarlo.

"Traducción de cosas simples como correos electrónicos, puede ser interpretación de llamadas telefónicas, conference calls, apoyo con visitas que vienen del extranjero para ayudarlos en reuniones". (Karina).

"La mayoría de los gerentes o socios que te piden esa ayuda, ellos saben inglés, saben inglés, y entienden perfectamente todo lo que el interlocutor les dice. Simplemente que nos piden la ayuda si no entienden una palabra, ellos quieren tenerte ahí para tener la seguridad que los están entendiendo". (Romina).

Como se puede evidenciar, las actividades que realiza un traductor van mucho más allá de solo traducir. Si bien no se puede generalizar, es posible afirmar que al traductor se les asigna actividades administrativas desde la comunicación interna hasta la redacción de documentos de mayor envergadura. 
(Varoma:2002). En el estudio de caso realizado por Anna Kuznik, la autora pudo evidenciar la multiplicidad de actividades que realiza el traductor.

"Defining the job content for the in-house translator, as visible in the translation of commercial correspondence, interpreting telephone conversations and secretarial activities..." (Kuznik, 2016, 226).

Respecto de la traducción propiamente dicha y de todas las actividades que se presentan en el proceso, el traductor cumple las funciones de terminólogo, documentalista, revisor, uniformizador y maquetador (este último en algunos casos). Los participantes crean sus propios glosarios y documentaciones, salvo un participante que, en casos extremos, en los que ya no se da abasto, recurre a un revisor externo (un hablante nativo de inglés) para que revise los documentos traducidos al inglés. Para los demás, no existe un revisor externo.

"El proceso de traducción es: yo traduzco, yo me autorreviso, y concluyo". (Reyna)

En cuanto a la interpretación, a excepción de un participante que solo se encarga de traducir, las modalidades más recurrentes son la consecutiva y la de enlace, dado que se realizan durante el acompañamiento al personal de la empresa en una visita o en una reunión o, por ejemplo, en el caso de las empresas de minería e industria, la presentación de maquinaria nueva por parte de los ingenieros u operarios. Son pocos los casos en los que se tienen que realizar interpretaciones simultáneas.

"Luego, como traductor es eso, como intérprete, también, como te decía, tienes actividades como interpretación de enlace, cuando vienen los técnicos tienes que seguirlos, tienes que acompañarlos todo el tiempo que estén en la planta, dentro". (María).

A partir de las últimas citas, se puede verificar la presencia de interpretación, si bien esta no es del tipo conferencias, se presenta en actividades propias de la empresa. 


\subsubsection{Ventajas y desventajas de ser traductor de planta}

Con respecto a las ventajas y desventajas de ser traductor de planta, los resultados fueron múltiples. En cuanto a las ventajas, los participantes consideraron que trabajar dentro de una empresa les brinda una estabilidad salarial, dado que todos los meses perciben un sueldo fijo. Asimismo, refirieron los beneficios sociales que perciben, como el seguro social, las vacaciones, la CTS (compensación por tiempo de servicio), las gratificaciones, entre otros. Además, estos beneficios representan una ventaja sustancial sobre los traductores freelance.

"Estoy en mejores condiciones, tengo todos mis beneficios, no solo nos dan vacaciones, nos dan nuestra compensación por tiempo de servicio (CTS), o sea aparte, recibimos bonos [...] Otra ventaja es que tienes un sueldo fijo todos los meses, y tienes beneficios laborales, tienes vacaciones, tienes gratificaciones, tienes pensión, tienes seguro". (Romina).

"Otra ventaja es que tienes un sueldo fijo todos los meses, y tienes beneficios laborales, tienes vacaciones, tienes gratificaciones, tienes pensión, tienes seguro.” (Romina).

"Lo bueno es que es un ingreso fijo, esa es la ventaja, como independiente puedes tener un mes fantástico, que la plata te puede durar por tres meses, pero después tienes un mes plano”. (Silvia).

Así como menciona Clara Gieure, muchos traductores buscan insertarse como traductores de planta dentro de una empresa tanto por la estabilidad económica como temporal que les ofrece. (Gieure: 2017). En comparación a los traductores freelance, por ejemplo, el ingreso no se rige a la cantidad de documentos que tienen que traducir, sino al sueldo que se estableció al momento de la contratación. Así, como se mencionaba en líneas anteriores, si hay temporadas con un mayor volumen de traducciones que otras, el sueldo no varía. 
Asimismo, gran parte de los participantes considera que el sector minero es el que ofrece mayores beneficios y además es el sector que más traductores contrata. Por otro lado, se tiene como ventaja la especialización que alcanzan los traductores, es decir, el perfeccionamiento y el incremento de conocimiento de la temática de un determinado sector. Por ejemplo, la participante que trabaja en minería se especializa en temas de minería, geología y, por ello, posee un gran dominio terminológico y temático.

"Tienes el dominio del tema específico que estás desarrollando, que estás traduciendo, dominio del rubro de sector que involucra estos temas específicos”. (Reyna).

“Te especializas de alguna manera en ese campo, aprendes, lo cual muchas veces no lo sabías antes. Entonces aprendes mucho y es una constante, el flujo de trabajo, la temática de trabajo que estás recibiendo, esa temática es casi siempre el mismo tema". (Silvia).

Otra ventaja a la que aludieron los participantes fue el hecho de tener a los especialistas a disposición para poder resolver sus dudas, a diferencia de los traductores que trabajan en agencias de traducción o como freelance.

"Entonces eso es superpositivo porque la persona que ha producido el texto y los especialistas en esa rama están ahí a tu alrededor, ese es el beneficio de trabajar en una empresa porque a veces cuando tú trabajas en un estudio (empresa de traducción), o trabajas freelance, no tienes el especialista a la mano". (Carolina).

"Un traductor de planta tiene toda la documentación paralela en terminología y especialistas a su mano". (Reyna).

Respecto de las desventajas, los participantes consideraron que, si bien tener un horario fijo es una ventaja, implica también una desventaja, dado que les limita el tiempo para poder realizar otras actividades. Y, por ello, el manejo del tiempo en comparación con el freelance no es de su libre albedrío. 
"Como freelance tienes tu tiempo a disposición, tú mismo regulas tu tiempo. El de agencia (traductor), bastante overtime, que te tienes que quedar. El de planta, tienes tiempos fijos, no tienes que quedarte fuera de tus tiempos, pero estás ajustado a los horarios de oficina que tengas que tener: nueve horas, ocho horas, igual es". (Juan).

Tal y como menciona Sofer (2012), los traductores de planta no tienen la flexibilidad ni la posibilidad de tiempo ni tampoco la posibilidad de cambiar a diferentes temas sobre los que puede traducir. Esta situación de llegar a lidiar con una temática en específico podría llevar al traductor a considerar su trabajo como repetitivo y poco desafiante.

Por otro lado, en lo que se refiere a la remuneración fija todos los meses, los traductores consideran que también es una desventaja, dado que el volumen de documentos a traducir no influye en el salario y que, en comparación con un freelance, estos últimos establecen sus propios precios.

"Independientemente del número de traducciones que hagas, tu sueldo es el mismo. Entonces, a diferencia de los freelance, tienen quizá un poco más de ventaja, que pueden cobrar por una cantidad de palabras, o siendo intérprete pueden cobrar por horas". (Carmen).

\section{Discusión}

partir de los datos recopilados a través de las encuestas, se puede concluir
que once de los catorce traductores se encuentran satisfechos con los
sueldos que reciben, y estos oscilan entre los 430 y 1450 dólares al mes, salvo
un caso, donde una participante ganó más de 1450 dólares. Asimismo, todos
los entrevistados consideran que la experiencia de haber trabajado como
traductor de planta fue gratificante, incluso para aquellos que consideraron que
su sueldo no era bueno. Esta situación implicaría que el principal beneficio que
reciben los traductores de planta no está necesariamente vinculado al dinero,
sino a otros factores que podrían ser la especialización, los beneficios sociales
o el aprendizaje continuo, por ejemplo. Del mismo modo, se puede ver que no
existe una diferencia abismal respecto del promedio general de los salarios.
Sin embargo, se debe tener en cuenta que los sueldos referidos no incluyeron 
los bonos o las gratificaciones que los traductores perciben como ingreso extra. Se considera que los beneficios pueden variar de acuerdo con la envergadura de cada empresa.

En cuanto a los contratos, se puede apreciar que hay a largo y corto plazo. La mayoría de los traductores entrevistados tienen contratos indefinidos. Hay algunos contratos que abarcan meses, pero cuentan con la posibilidad de renovación y, por ello, los traductores se encuentran trabajando durante un buen periodo con esas empresas.

Los resultados obtenidos a través de las entrevistas brindan un panorama más detallado, lo cual implica ser un traductor de planta. En primer lugar, la situación del traductor de planta en Lima es interesante. Todos los participantes coincidieron en que la profesión se encuentra muy poco difundida y, por ello, hay poco conocimiento y valoración de ella. La posición del traductor es relegada a la de otros trabajadores (Olohan, 2015), dado que, en muchas empresas, el cargo de traductor lo desempeña un profesional de otra carrera (Alejandro, 2012) o una secretaria bilingüe. Si bien esta investigación solo se ha centrado en los traductores de planta que son traductores profesionales, no se descarta la idea de que existan empresas que tengan como traductores de planta a profesionales bilingües de otras carreras. En Colombia, por ejemplo, los traductores no necesariamente han estudiado la carrera de Traducción e Interpretación para desempeñarse como tales. Muchos son empíricos que han estudiado otra carrera (Franco y Quiroz, 2013).

Las evidencias sobre la situación del traductor de planta se reflejan en la falta de un área específica para los traductores dentro de la empresa o en la falta de comprensión en la cantidad de tiempo que les lleva traducir. Sin embargo, este panorama cambia cuando los traductores comienzan a educar a los empleados de las diversas áreas de la empresa, es decir, hay cambios sustanciales cuando comienzan a explicarles qué es ser traductor, cuáles son sus implicancias y por qué es importante para el beneficio de la empresa. Esta situación es muy frecuente para muchos traductores, dado que la carrera en Perú es muy joven y son pocas las universidades que la imparten. Por otro lado, los participantes manifestaron que, luego de un tiempo, gran parte de los "clientes" comienzan a valorar su trabajo y las condiciones 
laborales tienden a mejorar. El traductor es, entonces, reconocido como una pieza central para la empresa (García, 2011).

Otro resultado resaltante fueron las competencias específicas que todo traductor de planta debe tener. Todos los participantes concluyeron que las competencias básicas son la lingüística, que se basa en el dominio de las lenguas de trabajo, en este caso, las preponderantes el español y el inglés (Franco y Quiroz, 2013) y la competencia traductora, que implica el procesamiento cognitivo necesario para satisfacer las demandas internas de traducción dentro de la empresa (Márquez, 2013). Por ello, los entrevistados necesitan realizar traducciones óptimas dentro de un contexto especializado a través de la implementación de todos los conocimientos lingüísticos y traductológicos (Clavijo et al., 2006). Para muchos traductores, este contacto con los conocimientos técnicos o especializados es un poco complicado al principio, dado que la formación que han recibido no se ha centrado en temáticas específicas como ingeniería o finanzas, por ejemplo. Sin embargo, estos traductores logran adaptarse muy rápidamente a una temática especializada gracias al desarrollo de su competencia traductora. Según Beeby et al. (2001), el desarrollo de esta competencia durante la formación académica le permite al traductor, no solo tener conocimientos en lenguas, sino también la habilidad de documentación, investigación y actitudinal para enfrentar diversas situaciones.

Del mismo modo, las habilidades blandas, entendidas como las escalas de valoración con las que una persona toma decisiones en su vida o reacciona frente a diversas situaciones y actitudes (Vargas y Vargas, 2016), se encuentran presentes en la labor del traductor de planta. Este último debe desarrollar la habilidad del control y manejo de emociones para trabajar con una diversidad de profesiones y lidiar con las presiones de los plazos de entrega frente a la demanda de encargos. Es muy importante que los traductores de planta tengan una relación cordial con los compañeros, puesto que así la probabilidad de que se generen problemas interpersonales es menor. En consecuencia, este hallazgo implica un cambio en la perspectiva que se tiene del traductor como un ser aislado y encerrado dentro de su oficina. Frente a esta red de contacto interpersonal, los traductores de planta requieren una habilidad blanda de flexibilidad, dado que necesitan adaptarse a diversas situaciones y requerimientos (Márquez, 2013). 
Por otro lado, en la mayoría de los casos, las presiones a las que se encuentran sujetos los traductores se relacionan con la priorización y los plazos de entrega de traducciones y el estrés que genera ser el único o uno de los pocos traductores de toda la empresa. Los participantes expresaron que traducen documentos de las diferentes áreas administrativas y de operaciones. Como menciona Berenzon (2004), los clientes dentro de una empresa se enfocan solo en el producto final y no en el proceso que debe llevar a cabo el traductor. Por ello, es vital que los clientes internos comprendan que los traductores no son sistemas de traducción automatizados y necesitan priorizar los encargos de acuerdo con las demandas internas. Se requiere mucha paciencia, control y orden para poder satisfacer a todos los pedidos.

Respecto de los resultados obtenidos sobre las actividades que realizan los traductores, todos se dedican a desarrollar otras tareas más allá de traducir (Kuznik, 2016). Si bien el nombre que recibe la actividad es "traducción de planta", la realidad es que implica otras actividades como interpretar, revisar textos, apoyo en la comunicación y algunas actividades secretariales (Kuznik, 2011). Por ejemplo, muchos participantes se encargan de contestar y recibir las llamadas de sus jefes cuando se tienen que comunicar en inglés. Además, todo el proceso de traducción que incluye la búsqueda terminológica, la documentación y la revisión es realizada por el traductor en su totalidad (Dam y Zethsen, 2009). A diferencia del estudio de Kuznik, los traductores no presentan un gestor de traducción interno (Kuznik, 2011), sino que son ellos quienes asumen todos los roles, desde gestor de proyectos hasta maquetadores.

Las interpretaciones, por su parte, no estuvieron exentas y se presentaron en diversas modalidades (Kuznik, 2016). Las más frecuentes son las consecutivas y las de enlace, ya sea en eventos internos o durante las visitas de personal extranjero. La modalidad simultánea no es muy frecuente.

Y el último resultado se enfoca en las ventajas y desventajas de ser un traductor de planta. Para los participantes, una de las ventajas más grandes es la estabilidad económica que les genera recibir un sueldo fijo todos los meses. Asimismo, los traductores entrevistados expresaron su satisfacción con los beneficios sociales, como la compensación por tiempo de servicio, los beneficios sociales, como el acceso a un seguro de salud, el aporte a un fondo de pensiones o a los beneficios económicos que cada empresa puede 
otorgar internamente. Del mismo modo, se tiene la ventaja de estar dentro de una planta junto con los especialistas de manera continua como fuente de conocimiento, y la posterior especialización en temas propios de la empresa (Gouadec, 1989). Asimismo, los participantes consideraron que es una ventaja tener un horario fijo, puesto que no requieren realizar horas extras para ganar más o para terminar los proyectos. Las actividades se realizan en la planta, y por ello, representa una ventaja frente a los freelance.

Por su parte, las desventajas que se encuentran al ser traductor de planta son la imposibilidad de cobrar por traducciones o número de palabras, lo cual haría que el salario varíe, y también la posibilidad de manejar los tiempos para poder llevar a cabo otras actividades (Sofer, 2012).

\section{Conclusiones}

Dor todo lo expuesto, se puede afirmar el presente estudio de caso es 1 una primera aproximación al mercado de la traducción de planta, dado que no existen investigaciones en el contexto latinoamericano o peruano que hayan estudiado esta temática. Por ello, se debe considerar que, al ser una aproximación, los resultados no deben generalizarse, sino que deben considerarse como parámetros que brindan una pequeña vista hacia el trabajo del traductor de planta y las implicancias del mismo. Asimismo, se considera que la presente investigación podría servir como un nuevo ámbito en los que los traductores pueden trabajar, y es distinto al de las empresas de traducción o al de los traductores freelance. 


\section{Fortalezas y limitaciones}

T a presente investigación tiene varias fortalezas resaltables. La primera Use encuentra en el carácter novedoso de la investigación de mercado vinculada a la traducción de planta en Lima, Perú. Por otro lado, se considera como una fortaleza el haber trabajado con los traductores profesionales, dado que fueron un grupo delimitado, del que se extrajo una gran cantidad de información determinante para el estudio.

Asimismo, otra ventaja fue trabajar con traductores que presentaban diferencias en los años de experiencia, dado que, de cierta manera, brindó la posibilidad de esbozar como va cambiando la imagen del traductor de planta con los años, cuáles son los desafíos y retos que los traductores que deseen trabajar en planta deberán afrontar. Por ejemplo, se tuvo que traductor con 23 años de experiencia solo se dedicaba a traducir. En su empresa no le pedían realizar interpretaciones. En cambio, a los más jóvenes se les encomienda muchas más labores, según el avance de la tecnología y demandas del mercado.

En lo que concierne a las limitaciones, se puede referir que la falta de disponibilidad total de tiempo no permitió contactar a un mayor número de traductores ni ampliar los sectores de investigación. Asimismo, excluir a los traductores empíricos no permitió realizar un contraste más profundo en lo que concierne a sus competencias ni brindar un panorama general de la traducción de planta en Lima, Perú. Por otro lado, tampoco se pudo contrastar la información, por ejemplo, con las perspectivas de los empleadores.

Si bien esta investigación es una aproximación, se considera que los resultados que ha brindado son útiles para el desarrollo de la profesión de traductor-intérprete en el mercado peruano. La imagen del traductor solitario encerrado en una oficina ha cambiado. Ahora es un ser multifacético que se encuentra en constante contacto con los demás, es una pieza importante dentro del engranaje empresarial. Por ello, se debe valorar su trabajo y difundirlo más allá de las fronteras peruanas. Este estudio de caso es un primer paso para investigaciones mucho más amplias. 


\section{Referencias}

Alejandro Candelero, E. (2002). Análisis de la necesidad de la enseñanza del lenguaje técnico petrolero en el programa de estudios de la licenciatura en idiomas con terminal en comunicación (Tesis de grado, Universidad Juárez Autónoma de Tabasco, México). Recuperado de https://goo.gl/R5EAvY

ASBANC (2014). Perú en el siglo XXI entre los líderes del crecimiento económico de la región. ASBANC Semanal, 93(4), 1-5. Recuperado de $\mathrm{http}: / /$ www.asbanc.com.pe/Publicaciones/n93.pdf\#search=Per\%C3\%BA\%20 en $\% 20$ el $\% 20$ siglo $\% 20 X X I \% 20$ entre $\% 201$ os $\% 201 \%$ C3\%ADderes $\% 20$ del $\% 20$ crecimiento $\% 20$ econ $\% \mathrm{C} 3 \% \mathrm{~B} 3 \mathrm{mico} \% 20 \mathrm{de} \% 201 \mathrm{a} \% 20 \mathrm{regi} \% \mathrm{C} 3 \% \mathrm{~B} 3 \mathrm{n}$

Beeby, A., Berenguer, L., Ensinger, D., Fox, O., Albir, A. H., Melis, N. M., ... y Presas, M. (2001). La competencia traductora y su adquisición. Quaderns: revista de traducción, 6, 39-45. Recuperado de https://goo.gl/eFFnqy

Benavides, B., Gálvez, A., Villanueva, I. y Garvich, M. (2015). Exploración a profundidad del mercado de traducción en Lima (Perú) (Tesis de grado, Universidad Peruana de Ciencias Aplicadas, Perú).

Berenzon, H. K. (2004). La formación del traductor en el lugar de trabajo. LETRAS, 1(36), 119-137. Recuperado de https://goo.gl/1ipSoM

Castillo, P., Montoro, C. y Tue, V. (2006). Hechos estilizados de la economía peruana. Banco Central de Reserva del Perú. Recuperado de https://goo. $\mathrm{gl} / \mathrm{bNFVSE}$

Choi, J. y Lim, H. O. (2000). An overview of the Korean translation market. Meta, 45(2), 383-392. http://dx.doi.org/10.7202/002235ar

Clavijo, B., Panqueva, M. C., Correal, M. C., Duque, M. I., Mendoza, E., Franco, C. y Galindo, J. A. (2006). Necesidad de la traducción en las empresas: procesos y perfiles. Revista Escuela de Administración de Negocios, 58, 61-78. Recuperado de https://goo.gl/C3a7ZT 
Dam, H. V. y Zethsen, K. K. (2009). Translators and (lack of) power-a study of Danish company translators' occupational status. Language at Work-Bridging Theory and Practice, 4(6). http://dx.doi.org/10.7146/law. v4i6.6189

Franco Bello, C. (2011). Traducción empresarial en el mundo globalizado de hoy. Revista EAN, 70, 132-146. Recuperado de https://goo.gl/WYfkbh

Franco Uribe, L. P. y Quiroz Herrera, G. (2013). Hacia un perfil profesional del traductor en Colombia. RevistaEAN,70. https://doi.org/10.21158/01208160. n70.2011.532

García Trujillo, W. H. (2011). Una propuesta que apunta a la integración de traducción y empresa. Revista Escuela de Administración de Negocios, 70, 68-77. Recuperado de https://goo.gl/VcG9Gt

Gonzáles de Olarte, E. (2001). La centralización económica del Perú: ¿problema o posibilidad? Actualidad Económica, 220, 19-21. Recuperado de https://goo.gl/WbUvom

Gieure, C. (2017) El traductor e intérprete emprendedor: análisis de la supervivencia de las empresas y los empresarios autónomos. Tesis Doctoral. Universidad de Alicante. Recuperado de https://rua.ua.es/dspace/ bitstream/10045/55629/1/tesis_clara_gieure_sastre.pdf

Gouadec, D. (1989). Le traducteur, la traduction et l'entreprise. AFNOR Tour Europe.

Gouadec, D. (2007). Translation as a profession. Ámsterdam: John Benjamins Publishing.

Kuznik, Anna (2010) El contenido de los puestos de trabajo de los traductores. El caso de los traductores internos en empresas de traducción en Barcelona. Tesis doctoral. Universitat Autònoma de Barcelona Recuperado de https:// ddd.uab.cat/pub/tesis/2011/hdl_10803_5279/ak1de1.pdf

Kuznik, A. (2011). Puestos de trabajo híbridos: cuatro indicadores del carácter heterogéneo de los puestos de trabajo internos en traducción. Sendebar, 22, 283-307. Recuperado de https://goo.gl/xAmRqu 
Kuznik, A. (2016). Work content of in-house translators in small and mediumsized industrial enterprises. Observing real work situations. JoSTrans. The Journal of Specialised Translation, 25(1), 213-231. Recuperado de https:// goo.gl/Bd9JDZ

Lozano Argüelles, C. (2011). Estudio sobre el mercado de la traducción en España (Tesis de grado, Universidad de Salamanca, España). Recuperado de https://goo.gl/1wWiX9

Márquez, D. A. (2013). Las once competencias del traductor: el perfil ideal en servicios de traducción. Comunicación, Cultura y Política, 4, 53-68. Recuperado de https://goo.gl/iHgljW

Medina, A. M., y Álvarez, C. (2014) La relación empresa-traducción en el sector agroalimentario andaluz. Skopos Revista Internacional de Traducción, 4, 187-206. Recuperado de https://www.uco.es/ucopress/ojs/ index.php/skopos/article/view/4364/4132

Ministerio de Trabajo y Promoción Social (2001). El empleo en el Perú: 19902000. Lima: Ministerio de Trabajo y Promoción Social.

Mora Rey, D. P. e Infante Martínez, M. (2012). El papel de los traductores en los tratados de libre comercio (Colombia-Estados Unidos) (Tesis de grado, Universidad EAN, Bogotá, Colombia). Recuperado de https://goo. $\mathrm{gl} / \mathrm{SwUjhb}$

Olohan, M. (2015). Scientific and technical translation. Londres: Routledge. Recuperado de https://goo.gl/GYUXZ4

Sofer, M. (2012). The global translator's handbook. Lanham: Rowman \& Littlefield. Recuperado de https://goo.gl/bj79Yq

Survey of the Canadian Translation Industry (14 octubre 2014). documents. $m x$. Recuperado de https://goo.gl/48HfYV

Vargas Hernández, M. y Vargas Hernández, M. (2016). Indicadores y metodología para la medición de competencias blandas. ITAL, CETICS. Recuperado de https://goo.gl/iA8zig 
